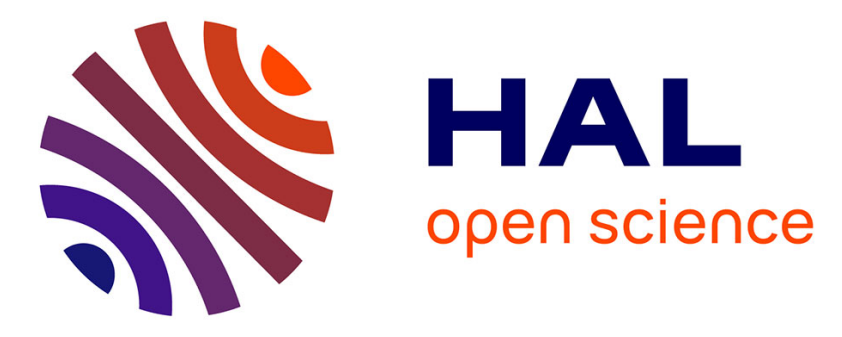

\title{
Generalization of the Rouard method to an absorbing thin film stack and application to surface plasmon resonance
}

Pierre Lecaruyer, Emmanuel Maillart, Michael Canva, Jannick Rolland

\section{- To cite this version:}

Pierre Lecaruyer, Emmanuel Maillart, Michael Canva, Jannick Rolland. Generalization of the Rouard method to an absorbing thin film stack and application to surface plasmon resonance. Applied optics, 2006, 45 (33), pp.8419-8423. 10.1364/AO.45.008419 . hal-00664485

HAL Id: hal-00664485

https://hal-iogs.archives-ouvertes.fr/hal-00664485

Submitted on 30 Jan 2012

HAL is a multi-disciplinary open access archive for the deposit and dissemination of scientific research documents, whether they are published or not. The documents may come from teaching and research institutions in France or abroad, or from public or private research centers.
L'archive ouverte pluridisciplinaire $\mathbf{H A L}$, est destinée au dépôt et à la diffusion de documents scientifiques de niveau recherche, publiés ou non, émanant des établissements d'enseignement et de recherche français ou étrangers, des laboratoires publics ou privés. 


\title{
Generalization of the Rouard method to an absorbing thin-film stack and application to surface plasmon resonance
}

\author{
Pierre Lecaruyer, Emmanuel Maillart, Michael Canva, and Jannick Rolland
}

\begin{abstract}
In the context of surface plasmon resonance (SPR) kinetic biochips, it is important to model the SPR phenomenon (i.e., extinction of reflectivity) toward biochip design and optimization. The Rouard approach that models reflectivity off a thin-film stack is shown to be extendable to any number of absorbing layers with no added complexity. Using the generalized Rouard method, the effect of SPR is simulated as a function of the wavelength for various metal thicknesses. Given an optimal metal thickness, the dependence of SPR on the angle of incidence and wavelength is also demonstrated. Such a model constitutes a potential basis for the efficient design and optimization of multidimensional sensors. (C) 2006 Optical Society of America

OCIS codes: $\quad 080.2720,310.6860,190.4350$.
\end{abstract}

\section{Introduction}

Maxwell's equations, together with the appropriate boundary conditions, determine the amplitudes and intensities of beams of light reflected off a thin-film stack. For a multiple-layer stack, Maxwell's equations are conventionally implemented using a matrix formulation such as first provided by Abeles, ${ }^{1}$ and further developed by Yeh. ${ }^{2-4}$ The Snell invariant is born from the continuity of the field components along each interface.

Building on the invariant obtained out of setting up Maxwell's equations, Rouard proposed another approach to the problem of modeling reflectivity off a multiple thin-film stack. ${ }^{5}$ The method is based on the computation of the resultant complex amplitude of the field reflected off the stack after ongoing multiple reflections within the stack. Inherent to the Rouard method is the generalization to as many layers as needed for an application without adding complexity. However, a key assumption of Rouard was to consider

The authors are with Laboratoire Charles Fabry de l'Institut d'Optique CNRS, Université Paris-Sud d'Orsay, bât.503 Centre Scientifique, 91403 Orsay cedex, France (pierre.lecaruyer@iota.upsud.fr). J. Rolland is also with the College of Optics and Photonics, University of Central Florida, Orlando, Florida 328162700 (jannick@odalab.ucf.edu).

Received 16 March 2006; revised 30 August 2006; accepted 1 September 2006; posted 7 September 2006 (Doc. ID 69023).

0003-6935/06/338419-05\$15.00/0

(C) 2006 Optical Society of America a stack with nonabsorbing layers. Although Rouard provided a different method for absorbing layers, such a method yields extremely convoluted expressions. ${ }^{5}$ Later, the Rouard method was described for various applications but still in a complex way. ${ }^{6,7}$

In this paper, we first briefly review the Rouard method, explicitly written for transparent media. We then discuss the generalization of this method to any number of absorbing layers. Finally, we apply the method to the computation of the specific case of surface plasmon resonance (SPR) and demonstrate its dependence on metal thickness, wavelength, and angle of incidence.

\section{Rouard Method for Modeling Reflectivity Off a Thin Dielectric Film Stack}

Let us first consider a simple stack including a substrate of infinite thickness (index of refraction $n_{1}$ ) and a thin film of thickness $e_{2}$, and an index of refraction $n_{2}$, as shown in Fig. 1. We shall assume, without loss of generality, that the thin film is in contact with a dielectric medium of infinite thickness and index of refraction $n_{3}$. A beam incident on the thin film via the substrate is divided into reflected and transmitted components, with new divisions each time the beam hits an interface. The beam reflected off layer 2 is then obtained by summing the multiple reflected beams. Because the reflectivity and transmissivity are functions of the state of polarization of the light, let us denote as $s$ the component of the electric field vector perpendicular to 


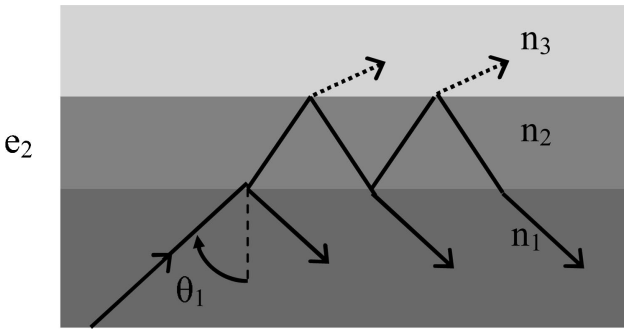

Fig. 1. (a) Schematic of a nonabsorbing thin film $\left(e_{2}, n_{2}\right)$ deposited between a glass substrate $\left(e_{1}, n_{1}\right)$ and a dielectric medium $\left(e_{3}, n_{3}\right)$; (b) schematic of a multiple layer stack with absorbing layers.

the plane of incidence (TE mode) and as $p$ the component of the electric field vector parallel to the plane of incidence (TM mode). With respect to the incident wave vectors $s$ and $p$, the relative complex amplitudes associated with the reflected and transmitted waves may be obtained using the Fresnel coefficients as ${ }^{6}$

$$
\begin{aligned}
& r_{1-2_{s}}=\frac{n_{1} \cos \theta_{1}-n_{2} \cos \theta_{2}}{n_{1} \cos \theta_{1}+n_{2} \cos \theta_{2}}, \\
& r_{1-2_{p}}=\frac{n_{1} \cos \theta_{2}-n_{2} \cos \theta_{1}}{n_{1} \cos \theta_{2}+n_{2} \cos \theta_{1}}, \\
& t_{1-2_{s}}=\frac{2 n_{1} \cos \theta_{1}}{n_{1} \cos \theta_{1}+n_{2} \cos \theta_{2}}, \\
& t_{1-2_{p}}=\frac{2 n_{1} \cos \theta_{1}}{n_{1} \cos \theta_{2}+n_{2} \cos \theta_{1}},
\end{aligned}
$$

where $\theta_{1}$ and $\theta_{2}$ represent the angles of incidence and refraction on the interface $1-2$. The reflectivities, defined as the ratios of reflected to incident energies, are given by

$$
R_{1-2 / p s}=r_{1-2 / p s}{ }^{2},
$$

and the transmissivities are given by

$$
T_{1-2 / p s}=\frac{n_{2} \cos \theta_{2}}{n_{1} \cos \theta_{1}} t_{1-2 / p s}^{2} .
$$

Equations (1)-(6) are useful in expressing the reflection and transmission at a single surface and are also shown to play a key role in multilayer computations.

By a simple summation of complex amplitudes, regardless of the polarization type, the reflected amplitude off layer 2 may be expressed as

$$
\begin{aligned}
r_{2}= & r_{1-2}+t_{1-2} e^{-j \varphi_{2}} r_{2-3} e^{-j \varphi_{2}} t_{2-1}+t_{1-2} e^{-j \varphi_{2}} r_{2-3} e^{-j \varphi_{2}} r_{2-1} \\
& \times e^{-j \varphi_{2}} r_{2-3} e^{-j \varphi_{2}} t_{2-1}+\cdots \\
= & r_{1-2}+\frac{t_{1-2} r_{2-3} t_{2-1} e^{-2 j \varphi_{2}}}{1-r_{2-1} r_{2-3} e^{-2 j \varphi_{2}}},
\end{aligned}
$$

where $r_{k-m}$ and $t_{k-m}$ are the Fresnel coefficients of reflection and transmission, respectively, between layers $k$ and $m$, and $\phi_{k}$ is the change in phase when propagating through layer $k$. Such a phase change is given by

$$
\varphi_{k}=\frac{2 \pi}{\lambda} n_{k} e_{k} \cos \theta_{k}
$$

where $\lambda$ is the wavelength, $n_{k}$ is the index of refraction of layer $k, e_{k}$ is its physical thickness, and $\theta_{k}$ is the associated term of the Snell's invariant. It can be shown using the Fresnel coefficients that

$$
t_{1-2} t_{2-1}=1-r_{1-2}^{2} .
$$

Thus most generally,

$$
r_{2}=\frac{r_{1-2}+r_{2-3} e^{-2 j \varphi_{2}}}{1+r_{1-2} r_{2-3} e^{-2 j \varphi_{2}}} .
$$

Let us now consider a multiple layer stack as shown in Fig. 2. Specifically, let us consider the layer of index of refraction $n_{4}$, thickness $e_{4}$, and associated Fresnel coefficient of reflection $r_{4-5}$. Layer 4 lies between layer 3 of index of refraction $n_{3}$ and layer 5 of index of refraction $n_{5}$. Similar to Eq. (10), the effective Fresnel coefficient for layer 4 is provided by

$$
r_{4}=\frac{r_{3-4}+r_{4-5} e^{-2 j \varphi_{4}}}{1+r_{3-4} r_{4-5} e^{-2 j \varphi_{4}}} .
$$

The reflected beam off layer 2 is then obtained by summing the multiple reflected beams.

Equation (11) summons up the multiple reflected beams off layer 4 . We can now consider the system from layer 4 to layer 5 to form an equivalent single layer of Fresnel coefficient $r_{4}$. The effective Fresnel coefficient of reflection from layer 3 and above is then given by

$$
r_{3}=\frac{r_{2-3}+r_{4} e^{-2 j \varphi_{3}}}{1+r_{2-3} r_{4} e^{-2 j \varphi_{3}}}
$$

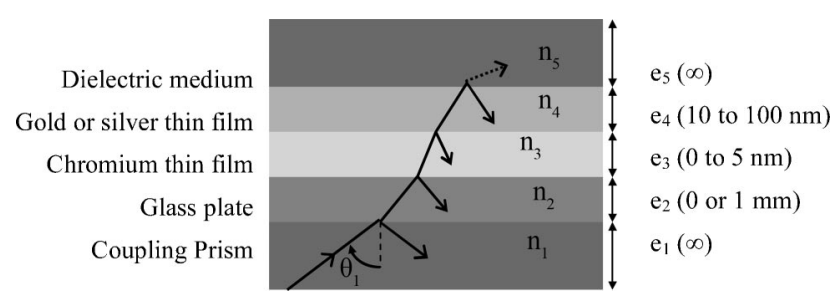

Fig. 2. Typical stack of multiple thin films and substrates of various thicknesses and refractive indices for surface plasmon resonance biosensors based on a Kretschmann configuration. 
Also,

$$
r_{2}=\frac{r_{1-2}+r_{3} e^{-2 j \varphi_{2}}}{1+r_{1-2} r_{3} e^{-2 j \varphi_{2}}}
$$

This expression is general as it applies to any angle of incidence, wavelength, and polarization with the appropriate Fresnel coefficients. Across multiple wavelengths, the reflectivity coefficients $r r^{*}$ sum up, where $r *$ is the complex conjugate of $r$.

\section{Generalization of the Rouard Method to Absorbing Layers}

In many experimental cases, some of the films are absorbing media, for example, in a five-layer problem, $n_{2}, n_{3}$, or $n_{4}$ will possess an imaginary part. Without loss of generality, let us consider a specific typical stack of layers for observing SPR. The layers going from the bottom to the top correspond to a coupling prism (1), a glass plate that acts as a substrate for the thin-film deposition (2), a chromium thin film (3), a silver or gold thin film (4), and a cover medium made of an aqueous buffer solution (5) (Fig. 2). The electromagnetic theory and the setting of Maxwell's equations together with proper continuity boundary conditions allow the Snell's law of refraction to be derived as

$$
\begin{aligned}
I & =n_{1} \sin \theta_{1}=n_{2} \sin \theta_{2}=n_{3} \sin \theta_{3}=n_{4} \sin \theta_{4} \\
& =n_{5} \sin \theta_{5},
\end{aligned}
$$

where, if any medium is absorbing, the corresponding index of refraction takes the form of a complex term. By replacing $n_{2}$ with $n_{2}=a_{n 2}+j b_{n 2}(a$ and $b$ being real values), we can write

$$
\sin \theta_{2}=\frac{n_{1} \sin \theta_{1}}{a_{n 2}+j b_{n 2}} .
$$

Since the prism is made of glass, $n_{1}$ is real, and we can deduce that $\sin \theta_{2}$ is complex. Thus a fundamental observation to this framework is realizing that, for an absorbing medium, both the index of refraction and the associated $\sin \theta_{2}$ term are complex. From the expression provided by Eq. (8), the product $n_{i} \cos \left(\theta_{i}\right)$ takes on a complex form, $a \pm j b$, as does $\cos \left(\theta_{i}\right)=$ $\left[1-\sin ^{2}\left(\theta_{i}\right)\right]^{1 / 2}$. In the case of an electromagnetic field of the form $e^{i \varphi}$, the positive sign of the real part of $\phi$ corresponds to an absorbing medium. Thus one cannot simply insert the index of refraction $n$ to be of the form $a_{n}+j b_{n}$ in the Fresnel coefficient. Instead, one must also set the Snell's law invariant of the proper form.

\section{Application of the Generalized Rouard Method to Demonstrating Surface Plasmon Resonance}

We illustrate the power of the extended Rouard method when evaluating the problem of modeling SPR. SPR has proven to be useful in the development of biosensors, specifically, in the determination of molecular interactions. ${ }^{7}$ The phenomenon of SPR emerges from the existence of a plasmon wave. ${ }^{8,9}$ The plasmon wave is an induced effect of the coupling between an electromagnetic field and the oscillation of a plasma. The term coupling means that, under a particular illumination condition characterized in part by the spectral characteristic of the source and the angle of incidence, all the energy of the beam is transferred to the plasmon wave. Hence the reflectivity of the incident beam is expected to drop down to zero in the case of $100 \%$ coupling. A drop peak will then appear on the reflectivity curve, where we would otherwise have expected total internal reflection with $100 \%$ reflectivity. Kretschmann proposed a prismmetal-air configuration to observe SPR, ${ }^{10}$ which we considered in our simulations. In such configuration, the electromagnetic field and the plasma oscillation were represented by the optical wave of an illuminating beam and the oscillation of the charge density of the metallic layer, respectively. Given a TM polarized collimated beam incident on the structure at a given angle of incidence and a wavelength that ensures the phase matching between the parallel component of the wavevector of the incident beam and the wavevector of the surface plasmon wave, the energy can be transferred from the light beam to the plasmon mode. No such transfer occurs for the TE mode.

In order to understand the parameter space and how SPR varies within such space, we establish SPR variation as a function of the metal thickness and the wavelength of the illuminating beam. Given optimal settings for the metal thickness and wavelength, we then investigate the variation of SPR as a function of the angle of incidence. Simulation of SPR as a function of these parameters allows guiding the development of biosensors.

\section{A. Surface Plasmon Resonance as a Function of Metal Thickness and Wavelength}

To clearly illustrate the influence of the thickness of the metallic layer on SPR, we consider a three-layer Kretschmann-type configuration, which was chosen to be composed of a metallic layer of silver located between a glass medium SF11 and an aqueous solution of water.

The curves represented in Fig. 3 report numerical results with silver films of various thicknesses at two different incident angles (i.e., $51^{\circ} 33^{\prime}$ and $60^{\circ} 42^{\prime}$ ). The spectral index curves of silver used to calculate the plasmon curves were obtained by smoothing spline interpolation of Sopra SA data. ${ }^{11}$ As expected, if there is no metallic layer, the system can be reduced to a SF11-water system. For the two chosen angles of incidence, the reflection must be total, which is confirmed by the spectral reflection curve equal to 1 through the visible spectrum. On the other end, if the film is too thick (i.e., $>140 \mathrm{~nm}$ ), the peak disappears and the system can be assimilated to a SF11-silver system with less than $100 \%$ reflectivity. In this case, an approximately constant reflectivity of the order of $80 \%-90 \%$ across the visible spectrum is predicted. For a few metal thick- 


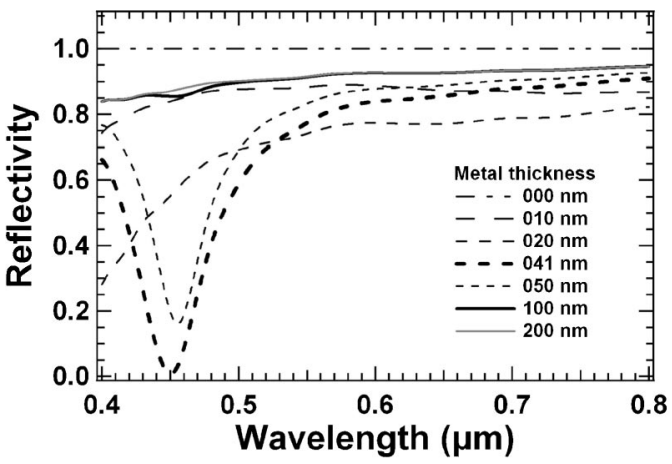

(a)

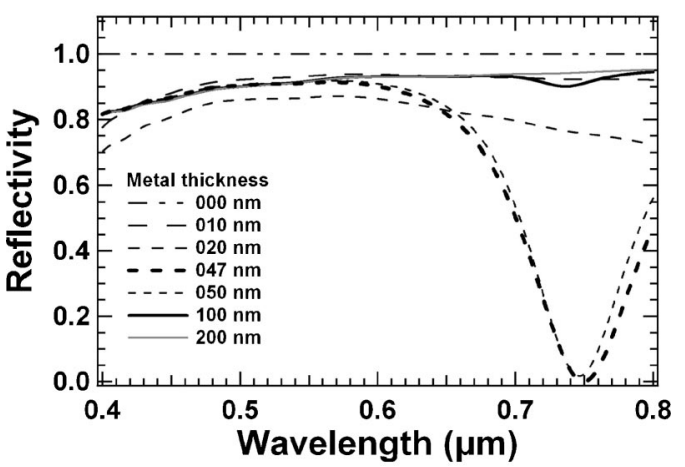

(b)

Fig. 3. (a) Variation of plasmon curves with wavelength and $\mathrm{Ag}$ metal thickness at angle of incidence (a) $60^{\circ} 42^{\prime}$ and (b) $51^{\circ} 33^{\prime}$.

nesses, whose values depend on the angle of incidence of the illuminating beam, the drop peak is deep, meaning that the incident energy is totally transferred to the plasmon wave, thus bringing the reflectivity down to zero. Such simulation illustrates the spectral plasmon coupling dependence for a given angle of incidence.

\section{B. Surface Plasmon Resonance as a Function of the Angle of Incidence}

In the prism-silver-water configuration, Fig. 4(a) shows the effect of the angle of incidence on the SPR as the angle of incidence is increased from $50^{\circ}$ and $70^{\circ}$ in the visible range. In Fig. 4(b), the reflectivity functions are presented as a function of the angle of incidence at two different wavelengths (i.e., Ag at $450 \mathrm{~nm}$ and $750 \mathrm{~nm}$ ).

The real part $n^{\prime}$ of the refractive index for silver can be shown to be almost constant across the visible spectrum, while the imaginary part $n^{\prime \prime}$ becomes twice as large as the wavelength increase from $450 \mathrm{~nm}$ to $750 \mathrm{~nm}$. This variation of $n^{\prime \prime}$ thus explains the narrowing of the peak of absorption as the imaginary part gets larger.

\section{Discussion}

In our field of SPR sensors, in view of gaining sensitivity, we currently apply such calculation to the optimization of multilayered structures and to determining the technological requirements concern-

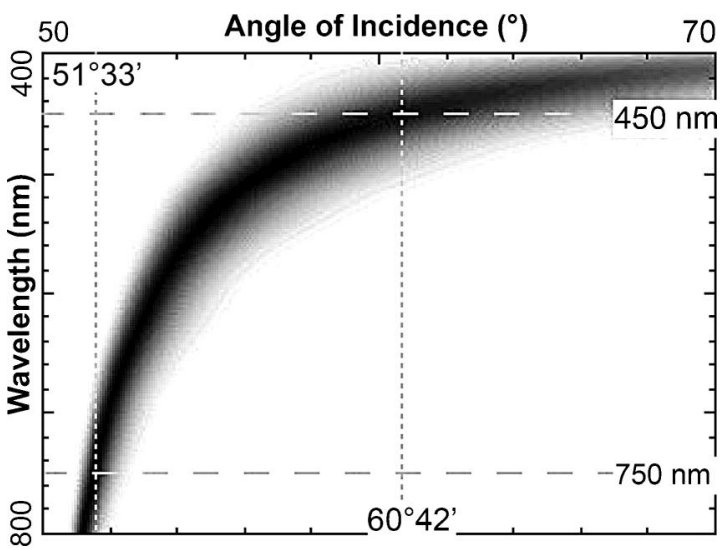

(a)

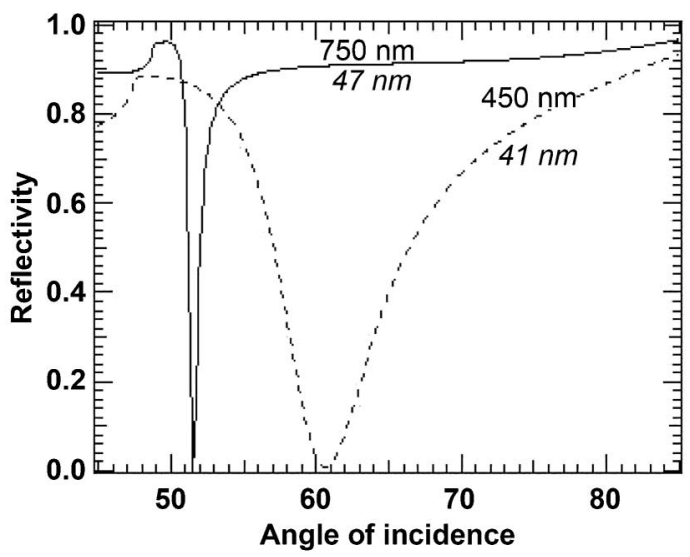

(b)

Fig. 4. Reflectivity surface plot of the configuration SF11-Agwater (a) in $3 \mathrm{D}$ between $50^{\circ}$ and $70^{\circ}$ in the visible range and (b) in $2 \mathrm{D}$ as a function of the angle of incidence for $450 \mathrm{~nm}$ and $750 \mathrm{~nm}$ for the optimized thicknesses.

ing the accuracy and homogeneity of the different films and materials.

This model is also used to quantify the biomolecular interactions that take place on our biochip surface.

\section{Conclusion}

The Rouard method, originally proposed to model the reflectivity of a multiple nonabsorbing layer stack, was shown to be applicable with proper handling of the Snell invariant and the complex index of refraction to the problem of modeling reflectivity for a multiple absorbing layer stack. The advantage of the approach lies in its simple implementation and generalization to as many layers as necessary by means of recursive loops.

The generalized Rouard method was then successfully applied to the problem of modeling SPR and particularly to the computation of reflectivity through a Kretschmann configuration. The variations of SPR with the thickness of the metal layer, the wavelength, and the angle of incidence were demonstrated. This investigation provides a potential basis for the efficient design of biosensors. 
This work was funded by the Centre National de la Recherche Scientifique (CNRS), a French government grant, and the Florida Photonics Center of Excellence. The authors acknowledge fruitful discussion with Yves Lévy.

\section{References}

1. F. Abeles, "Recherches sur la propagation des ondes électromagnétiques sinusoïdales dans les milieux stratifiés. Application aux couches minces," Ann. Phys. (Paris) 5, 596-640 (1950).

2. P. Yeh, Optical Waves in Layered Media (Wiley, 1988), p. 406.

3. P. W. Baumeister, Optical Coating Technology (SPIE, 2004).

4. H. A. McLeod, Thin Film Optical Filters, 3rd ed. (Institute of Physics, 2002).

5. M. P. Rouard, "Etudes des propriétés optiques des lames métalliques très minces,” Ann. Phys. (Paris) Ser. II 7, 291-384 (1937).

6. K. C. Park, "The extreme values of reflectivity and the condi- tions for zero reflection from thin dielectric films on metal," Appl. Opt. 3, 877-881 (1964).

7. Y. Dankner and A. Katzir, "Evanescent wave spectroscopy for the determination of the optical constants of thin silver films deposited on a silver-halide fiber," Appl. Opt. 36, 873-876 (1997).

8. M. Born and E. Wolf, Principles of Optics: Electromagnetic Theory of Propagation, Interference and Diffraction of Light (Pergamon, 1959).

9. B. Rothenhausler and W. Knoll, "Surface plasmon microscopy," Nature 332, 615-617 (1988).

10. U. Fano, "The theory of anomalous diffraction gratings and of quasi-stationary waves on metallic surfaces (Sommerfeld's waves)," J. Opt. Soc. Am. 31, 213-222 (1941).

11. A. Otto, "Excitation of surface plasma waves in silver by the method of frustrated total reflection," Z. Physik 216, 398-410 (1968).

12. E. Kretschmann and H. Raether, "Radiative decay of nonradiative surface plaasmons excited by light," Z. Naturforsch 23A, 2135-2136 (1968).

13. Available: http://www.sopra-sa.com/index2.htm. 